\title{
riccafd
}

Revista Iberoamericana de Ciencias de la Actividad Física y el Deporte

\section{ACTIVIDAD FÍSICA EN EL TIEMPO LIBRE PREVIENE ENFERMEDADES CARDIACAS/CARDIOVASCULARES: UNA REVISIÓN SISTEMÁTICA}

\section{PHYSICAL ACTIVITY DURING LEISURE TIME PREVENTS CARDIAC/CARDIOVASCULAR DISEASE: A SYSTEMIC REVISION}

Romero-Barquero Cecilia Enith ${ }^{1 \mathrm{ABCF}}$

${ }^{1}$ Romero Barquero, C. E. Doctora en Ciencias del Movimiento Humano, Profesora universitaria, Escuela de Educación Física y Deportes, Universidad de Costa Rica, Costa Rica, cecilia.romero@ucr.ac.cr

\author{
Responsabilidades \\ A Diseño de la investigación \\ ${ }^{\mathrm{B}}$ Recolector de datos \\ ${ }^{\mathrm{C}}$ Redactor del trabajo \\ D Tratamiento estadístico \\ E Apoyo económico \\ F Idea original y coordinador de toda la investigación
}

Correspondencia: Cecilia Enith Romero Barquero cecilia.romero@ucr.ac.cr Recibido el 5 de febrero de 2019

Aceptado el 17 de julio de 2019

DOI: http://dx.doi.org/10.24310/riccafd.2020.v9i1.8296

\section{RESUMEN}

La presente revisión sistemática examina la incidencia de la actividad física realizada en el tiempo libre (AFTL), en la prevención del riesgo y mortalidad por enfermedades cardiacas y cardiovasculares. Las AFTL reportadas en cada uno de los 29 estudios observacionales seleccionados, se clasificaron de acuerdo con los tipos de actividades recreativas que señala la literatura. Por lo tanto, luego del análisis surgieron cuatro categorías: recreación física, recreación artística, recreación al aire libre y recreación de pasatiempos. Asimismo, se establece que las AFTL, y en especial las de alta intensidad como las de recreación física y al aire libre, contribuyen a prevenir los factores de riesgo y mortalidad por enfermedad cardiovascular y cardiaca. Para futuras investigaciones, es importante incorporar la clasificación de actividades recreativas y analizar la influencia que cada tipo de estas actividades puede tener en la prevención de enfermedades cardiovasculares o cardiacas coronarias. 
Palabras clave: actividades físicas, enfermedades coronarias, enfermedades cardiovasculares, riesgo, mortalidad, tiempo libre, recreación.

\begin{abstract}
This systematic review examines the incidence of physical activity during leisure time (PALT), in the prevention of risk and mortality from cardiac and cardiovascular disease. The PALT reported in each of the 29 observational studies selected, were classified according to the types of recreational activities present in the literature. Therefore, after the analysis, four categories emerged: physical recreation, artistic recreation, outdoor recreation and recreation due to hobbies. Likewise, it is established that PALT, especially those of high intensity such as physical and outdoor recreation, contribute to prevent risk factors and mortality due to cardiovascular and cardiac disease. For future research, it is important to incorporate the classification of recreational activities and analyze the influence that each type of these activities can have on the prevention of cardiovascular and cardiac coronary disease.
\end{abstract}

Key Words: physical activities, coronary disease, cardiovascular disease, risk, mortality, leisure time, recreation.

\title{
INTRODUCCIÓN
}

El sedentarismo es un problema constante en las personas de todas las edades, por lo que profesionales de la salud insisten que la práctica constante de actividad física (AF) es necesaria para mantener estilos saludables de vida. Asimismo, la inactividad física influye en la aparición de enfermedades y perjudica la calidad de vida (1). Por ejemplo, en los últimos años la incidencia en enfermedades cardiovasculares (ECV) ha venido en aumento. Según la Organización Mundial de la Salud (OMS), las ECV son la principal causa de muerte en todo el mundo. En el 2008 murieron 17,3 millones de personas (30\% de todas las muertes registradas en el mundo), de esas muertes 7,3 millones se debieron a enfermedades cardiacas coronarias (ECC). Se estima que para el año 2030 fallezcan cerca de 23,6 millones de personas por ECV. Además, de acuerdo a los informes de la OMS, las ECV afectan a ambos sexos por igual y cerca del $80 \%$ se produce en países en vías de desarrollo (2). Un ejemplo de ello es Costa Rica, país en el que diariamente mueren 13 personas en promedio por esta causa (3).

Existen varios factores de riesgo cardiovascular que son modificables, una manera de revertirlos es por medio de la práctica regular de AF (4). De acuerdo con el American College of Sports Medicine (ACSM), la AF es "cualquier movimiento corporal producido por los músculos esqueléticos que provocan un gasto energético, por encima de los niveles de reposo basal" (5, p.1337). Entre las AF se encuentra el ejercicio, los deportes y las actividades físicas realizadas como parte de la vida diaria, la ocupación, el ocio y el transporte activo (5). En esta revisión, se hizo especial énfasis en buscar estudios que trataran la práctica de 
la actividad física en el tiempo libre (AFTL). Entendiendo por tiempo libre, como aquel en el que las personas no se dedican al trabajo, ni al estudio, ni a labores hogareñas (6).

Entre las principales actividades que se hacen en el tiempo libre se encuentran las de ocio o recreativas. Éstas se ejecutan y seleccionan de manera voluntaria, favorecen la salud, producen gozo, diversión, placer y desarrollo integral en la población que las practica. Dichas actividades se clasifican en recreación: 1) física (deportes, juegos y actividades físicas), 2) artística, 3) social, 4) al aire libre, 5) cognitiva, 6) de enriquecimiento y actualización personal, 7) pasatiempos, 8) turismo y 9) espiritual (7).

La práctica de la AF es importante, pero también se debe cumplir con ciertos parámetros para que sea idónea y eficaz para la salud. De acuerdo con la OMS, las personas de 5 a 17 años deben realizar AF (juegos, deportes, desplazamientos, actividades recreativas, educación física o ejercicios programados, en el contexto de la familia, la escuela o comunidad), al menos 60 minutos diarios continuos o en intervalos y a intensidades de moderada a vigorosa, lo cual colabora a desarrollar un aparato motor y un sistema cardiovascular sano, entre otros beneficios (2).

En cuanto a las personas de 18 a 64 años se recomienda realizar al menos 150 minutos semanales de AF aeróbica, de intensidad moderada, o bien 75 minutos de AF aeróbica vigorosa cada semana. La realización de la práctica aeróbica en cada sesión debe tener como mínimo 10 minutos continuos. En el caso de personas mayores de 65 años las recomendaciones son similares, además de incluir actividades que mejoren su equilibrio para evitar caídas $(2,5)$.

Los parámetros para realizar AF se encuentran claramente definidos, pero se vuelve interesante estudiar el impacto que cada tipo de AF (de desplazamiento o transporte, en el hogar, la ocupacional y en el tiempo libre) causa en la salud cardiovascular, en especial la AFTL, cuyo estudio ha venido en crecimiento (8, 9). Consecuentemente, en la presente revisión sistemática se establecen las siguientes preguntas: ¿cuál es la incidencia que la AFTL puede tener para contrarrestar los riesgos y la mortalidad por ECV y ECC?, ¿qué tipo de AFTL se reporta en los estudios, según la clasificación en recreación?, ¿existe diferencia en los resultados, de acuerdo al tipo e intensidad de las AFTL? y por último, ¿qué recomendaciones se pueden ofrecer a la población en general, según los resultados de los diferentes estudios analizados?, estas son las interrogantes que se pretenden aclarar por medio del análisis de varios artículos de investigación que examinaron la práctica de AFTL o "leisure time" y que a su vez, han sido clasificadas según los tipos de actividades recreativas (AR).

\section{OBJETIVOS}

1. Clasificar las AFTL reportadas en los estudios seleccionados, según los tipos de actividades recreativas (AR) que establece la literatura. 
2. Describir la incidencia que la AFTL, y en específico las recreativas, tiene en la prevención de la mortalidad y de los riesgos de las ECV y ECC, según el tipo e intensidad de la AR.

3. Detectar en los estudios recomendaciones para el fomento de la práctica de las AFTL, que permitan la prevención o disminución de los factores de riesgo y mortalidad por ECV y ECC.

\section{MATERIAL Y METODOS}

La presente revisión sistemática da a conocer los efectos encontrados en una serie de estudios vinculados con la AFTL y su relación con prevención de la mortalidad y de los riesgos de ECV y ECC. Para esta revisión se utilizó la base de datos de SPORTDiscus y Medline Complete. Para la búsqueda de las referencias se usó el término: leisure time, asociado a los términos physiological benefit, physiological effect, physical activity, mortality, cardiovascular health, coronary heart disease y cardiovascular risk factors, se encontraron 3073 artículos.

Después del filtro con las palabras claves se procedió a seleccionar los artículos. Los criterios de inclusión fueron: 1) artículos que evaluaron los efectos generados por la participación en AFTL, 2) efectos a nivel físico - fisiológico,

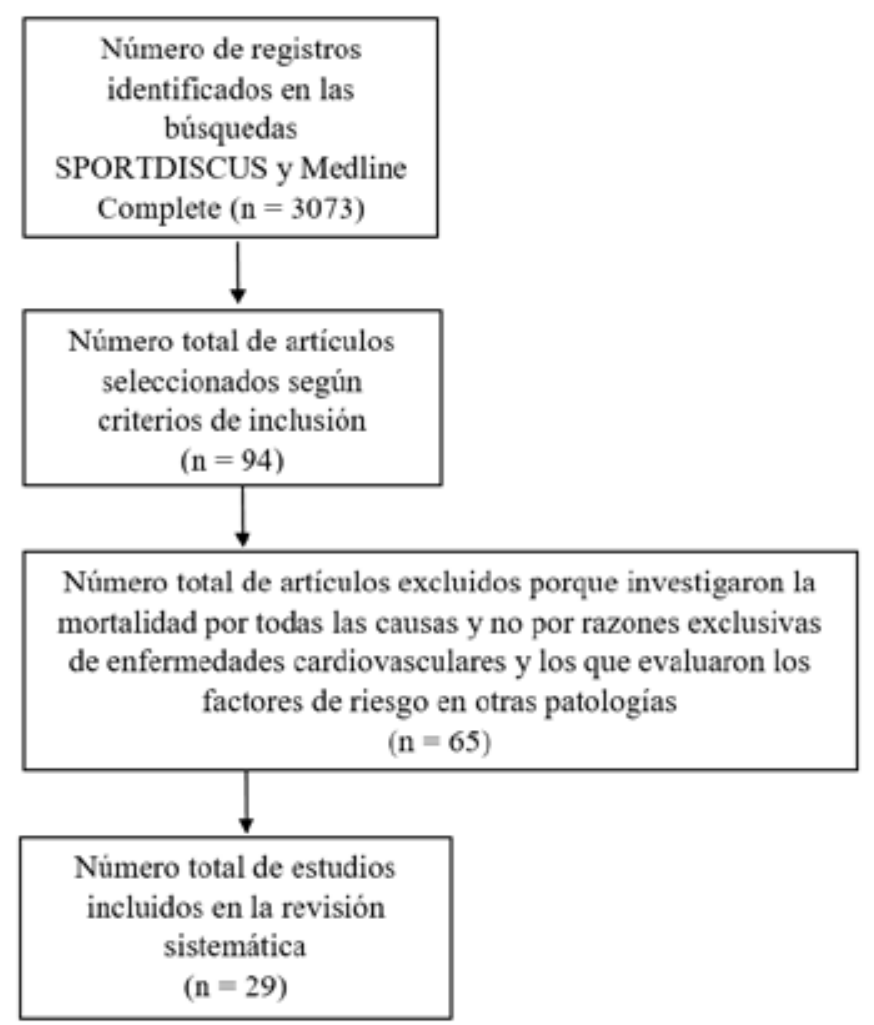

Figura 1. Diagrama de flujo del proceso de selección de los artículos. Fuente: Elaboración propia. 
en relación con la mortalidad y/o riesgos de ECV y ECC, 3) personas desde adolescentes hasta adulta mayor, de ambos sexos, físicamente sanos o con alguna patología, 4) publicación en inglés o español, 5) publicación hasta noviembre de 2018. De esta búsqueda se seleccionaron 94 estudios potenciales. De ellos, se excluyeron los que investigaron la mortalidad por todas las causas y no por razones específicas de ECV y ECC, los que evaluaron los factores de riesgo en otro tipo de patología y los que no eran estudios observacionales. Por lo tanto, 29 artículos formaron parte de las referencias de esta revisión. La Figura 1 presenta un diagrama del proceso de búsqueda y selección que se realizó en este estudio.

\section{RESULTADOS}

De los 29 artículos seleccionados, 15 presentan los resultados de participar en AFTL con respecto a los factores de riesgo de ECV y/o ECC (Ver Tabla 1). Los otros 14 estudios muestran los hallazgos en la prevención de la mortalidad por ECV y/o ECC, producto de la participación en AFTL (Ver Tabla 2). En ambas tablas, se indica el tipo e intensidad de las AFTL que fueron parte de cada una de las investigaciones analizadas.

Tabla 1. Estudios que examinan la relación entre el tipo y la intensidad de la actividad física realizada en el tiempo libre y el riesgo de enfermedad cardiaca coronaria y cardiovascular.

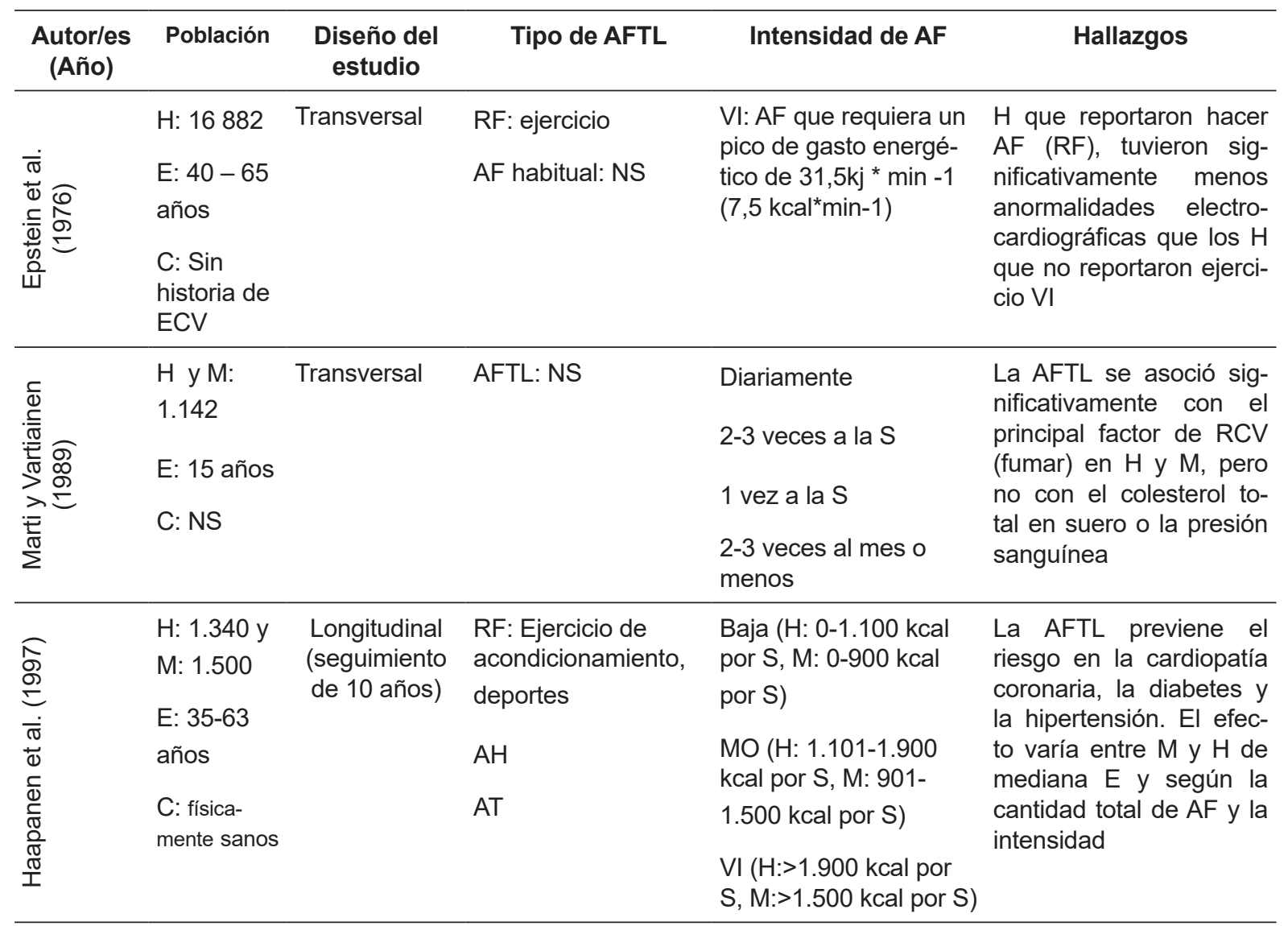




\begin{tabular}{|c|c|c|c|c|c|}
\hline $\begin{array}{c}\text { Autor/es } \\
\text { (Año) }\end{array}$ & Población & $\begin{array}{l}\text { Diseño del } \\
\text { estudio }\end{array}$ & Tipo de AFTL & Intensidad de AF & Hallazgos \\
\hline 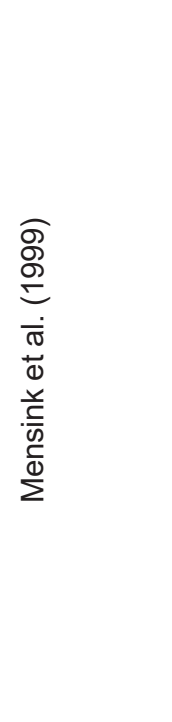 & $\begin{array}{l}\text { H: } 4.942 \text { y } \\
\text { M: } 5.885 \\
\text { E: } 50-69 \\
\text { años } \\
\text { C: sin his- } \\
\text { toria previa } \\
\text { de ECV, } \\
\text { cerebro- } \\
\text { vascular o } \\
\text { diabetes }\end{array}$ & Transversal & $\begin{array}{l}\text { RF, RP, RA, RAL: } \\
\text { ir de compras, } \\
\text { caminar, ciclismo, } \\
\text { gimnasia (LI), campo } \\
\text { traviesa, escalar, } \\
\text { bailar, ballet, bailar } \\
\text { Jazz, rutinas de } \\
\text { gimnasio y aeróbi- } \\
\text { cas, patinar, fútbol, } \\
\text { nadar, baloncesto, } \\
\text { voleibol balonmano, } \\
\text { esquí alpino, tenis } \\
\text { de mesa, bádminton, } \\
\text { ejercicios en la casa, } \\
\text { correr largas distan- } \\
\text { cias, boxeo, lucha, } \\
\text { artes marciales, } \\
\text { judo, karate, tenis, } \\
\text { squash (MO y VI) }\end{array}$ & $\begin{array}{l}\text { LI: } 3 \text { - 4,5 MET } \\
\text { MO y VI: } 5 \text { - } 9 \text { MET o } \\
\text { por frecuencia (de } 2 \text { a } \\
12 \text { veces al mes, de } 3 \\
\text { a } 5 \text { veces a la S o más, } \\
0,5 \text { - } 2 \text { h semanales) }\end{array}$ & $\begin{array}{l}\mathrm{MO} \text { y } \mathrm{VI} \text {, o una conduc- } \\
\text { ción frecuente de LI de } \\
\mathrm{RF}-\mathrm{RP} \text { - RAL- RA puede } \\
\text { tener un beneficio sus- } \\
\text { tancial para el perfil de } \\
\text { RCV en adultos mayores } \\
\text { Mayor frecuencia y ma- } \\
\text { yor duración de AFTL } \\
\text { contribuye a una mayor } \\
\text { reducción de los factores } \\
\text { de RCV }\end{array}$ \\
\hline 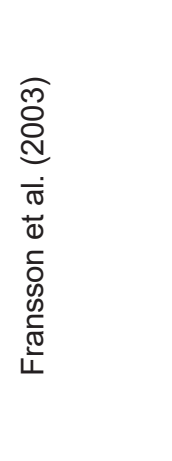 & $\begin{array}{l}\text { H: } 7.168 \text { y } \\
\text { M: } 3.245 \\
\text { E: } 19-70 \\
\text { años } \\
\text { C: NS }\end{array}$ & Transversal & $\begin{array}{l}\text { AFTL: NS } \\
\text { AO } \\
\text { AH }\end{array}$ & $\begin{array}{l}\text { Nunca o rara vez } \\
\text { Algunas veces } \\
\text { De manera regular }\end{array}$ & $\begin{array}{l}\text { La AFTL y la AF total (AO } \\
\text { y AH) se asociaron inver- } \\
\text { samente con los factores } \\
\text { de RCV, especialmente } \\
\text { con el HDL, en H y M } \\
\text { La AFTL se asoció be- } \\
\text { neficiosamente con los } \\
\text { niveles plasmáticos de fi- } \\
\text { brinógeno y de HDL en H } \\
\text { y M y con la hipertensión } \\
\text { en los H }\end{array}$ \\
\hline 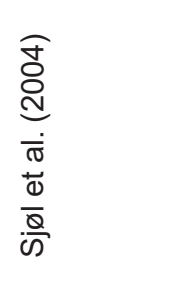 & $\begin{array}{l}\mathrm{H}: 4.322 \text { y } \\
\text { M: } 4.322 \\
\text { E: } 30-60 \\
\text { años } \\
\text { C: NS }\end{array}$ & $\begin{array}{l}\text { Longitudinal } \\
\text { (seguimiento } \\
\text { de } 28 \text { años) }\end{array}$ & $\begin{array}{l}\text { RF: caminar y activi- } \\
\text { dades similares } \\
\text { Deportes competi- } \\
\text { tivos }\end{array}$ & $\begin{array}{l}\text { Sedentarios } \\
\text { Caminar y actividades } \\
\text { similares } \\
\text { Actividades regulares } \\
\text { Deportes competitivos }\end{array}$ & $\begin{array}{l}\text { Un IMC elevado y AFTL } \\
\text { sedentarias provoca un } \\
\text { aumento en ECV }\end{array}$ \\
\hline 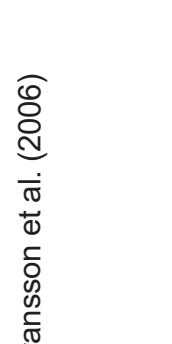 & $\begin{array}{l}\text { H: } 1.204 \text { y } \\
\text { M: } 550 \\
\text { E: } 45-70 \\
\text { años } \\
\text { C: casos } \\
\text { de infarto }\end{array}$ & $\begin{array}{l}\text { Estudio de } \\
\text { caso-control }\end{array}$ & $\begin{array}{l}\text { RF: caminatas oca- } \\
\text { sionales, ejercicio (al } \\
\text { menos } 30 \text { minutos y } \\
\text { que los hiciera que- } \\
\text { dar sin aliento) }\end{array}$ & $\begin{array}{l}\text { Muy poco ejercicio y } \\
\text { caminatas ocasionales } \\
\text { Ejercicio de vez en } \\
\text { cuando, al menos una } \\
\text { vez a la S } \\
\text { Ejercicio dos o más } \\
\text { veces a la S }\end{array}$ & $\begin{array}{l}\text { La AFTL de manera re- } \\
\text { gular disminuye el riesgo } \\
\text { de IM en } 38 \% \text { - } 46 \% \text {, en } \\
\text { personas de peso magro } \\
\text { (delgadas), normal y con } \\
\text { sobrepeso, comparado } \\
\text { con ser sedentario, pero } \\
\text { no en personas obesas }\end{array}$ \\
\hline
\end{tabular}




\begin{tabular}{|c|c|c|c|c|c|}
\hline $\begin{array}{l}\text { Autor/es } \\
\text { (Año) }\end{array}$ & Población & $\begin{array}{l}\text { Diseño del } \\
\text { estudio }\end{array}$ & Tipo de AFTL & Intensidad de AF & Hallazgos \\
\hline 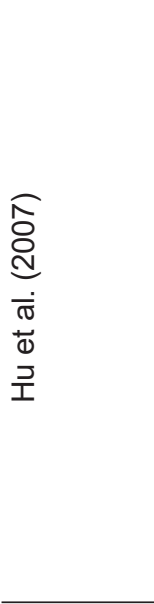 & $\begin{array}{l}\text { H y M: } \\
41.053 \\
\text { E: } 25-64 \\
\text { años } \\
\text { C: sanos } \\
\text { físicamente } \\
\text { (sin historia } \\
\text { de ECC } \\
\text { e infarto } \\
\text { cerebral }\end{array}$ & $\begin{array}{l}\text { Longitudinal } \\
\text { descriptivo } \\
\text { (seguimiento } \\
\text { de } 10 \text { años) }\end{array}$ & $\begin{array}{l}\text { AT: en bicicleta o } \\
\text { caminando } \\
\text { RF: montar en bi- } \\
\text { cicleta, gimnasia, } \\
\text { natación entre otros } \\
\text { RA: baile } \\
\text { RAL: pasear, jardi- } \\
\text { nería } \\
\text { RP: leer, ver la tele- } \\
\text { visión } \\
\text { AO }\end{array}$ & $\begin{array}{l}\text { AFTL: LI: leer, ver tele- } \\
\text { visión o hacer alguna } \\
\text { AF menor, MO: AF } \\
\text { más de } 4 \text { hrs / S, como } \\
\text { caminar, montar en bi- } \\
\text { cicleta, jardinería y VI: } \\
\text { más de } 3 \text { hrs/ S, como } \\
\text { correr, trotar, nadar o } \\
\text { deportes competitivos } \\
\text { varias veces a la S }\end{array}$ & $\begin{array}{l}\text { MO y VI de AO, RF y RAL } \\
\text { en H y M, así como AT en } \\
\text { M, se asoció con un ries- } \\
\text { go reducido de eventos } \\
\text { de ECC en } 10 \text { años }\end{array}$ \\
\hline 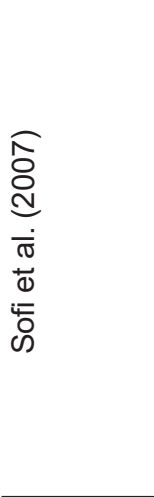 & $\begin{array}{l}\text { H: } 365 \text { y } \\
\text { M: } 567 \\
\text { E: } 54 \text { años } \\
\text { en prome- } \\
\text { dio } \\
\text { C: NS }\end{array}$ & Transversal & $\begin{array}{l}\text { RF: ciclismo, ca- } \\
\text { minar por placer y } \\
\text { trabajos de hágalo } \\
\text { usted mismo, gim- } \\
\text { nasia, correr, jugar } \\
\text { fútbol, golf, tenis, } \\
\text { natación } \\
\text { RAL: jardinería } \\
\text { RA: bailar }\end{array}$ & $\begin{array}{l}\text { Ausente o LI: inactivo o } \\
\text { caminata de } 30 \text { minu- } \\
\text { tos, menos de } 3 \text { veces } \\
\text { a la S } \\
\text { MO: caminatas de } 30 \\
\text { minutos de } 3 \text { a } 5 \text { veces } \\
\text { a la S o ejercicios de- } \\
\text { portivos } 1 \text { vez a la S } \\
\text { VI: actividades depor- } \\
\text { tivas al menos } 2 \text { veces } \\
\text { a la S }\end{array}$ & $\begin{array}{l}\text { AFTL de MO o VI se aso- } \\
\text { ció de manera significati- } \\
\text { va con un perfil de RCV } \\
\text { más favorable en térmi- } \\
\text { nos de antropometría, } \\
\text { parámetros metabólicos } \\
\text { y lipídicos }\end{array}$ \\
\hline 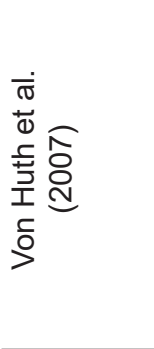 & $\begin{array}{l}\text { H: } 3.302 \text { y } \\
\text { M: } 3.482 \\
\text { E: } 30-60 \\
\text { años } \\
\text { C: NS }\end{array}$ & Transversal & $\begin{array}{l}\text { AT: bicicleta, caminar } \\
\text { o correr } \\
\text { RF: caminar, andar } \\
\text { en bicicleta } \\
\text { RAL: jardinería } \\
\text { AFT }\end{array}$ & $\begin{array}{l}\text { AT: menos de } 15 \text { min, } \\
\text { 15-30 min, } 30 \text { min a } 1 \\
\text { hora; } 1 \mathrm{~h} \text { o más } \\
\text { RF-RAL: } 0 \text { min; aproxi- } \\
\text { madamente } 1 / 2 \mathrm{~h} / \mathrm{S} ; \\
\text { aprox. } 1 \mathrm{~h} / \mathrm{S} \text {; aprox. } \\
\text { 2-3horas/S; aprox.4-6 } \\
\text { h/S; y } 7 \mathrm{~h} / \mathrm{S} \text { o más }\end{array}$ & $\begin{array}{l}\text { AT, RF-RAL y AFT se } \\
\text { asoció positivamente con } \\
\text { el HDL y negativamente } \\
\text { con el LDL, los triglicéri- } \\
\text { dos, la CC y el IMC }\end{array}$ \\
\hline 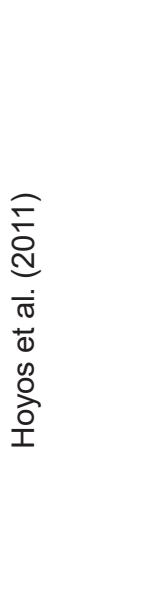 & $\begin{array}{l}\text { M: } 288 \text { y } \\
\text { H: } 111 \\
\text { E: } 19,74 \\
\text { años en } \\
\text { promedio } \\
\text { C: NS }\end{array}$ & Transversal & $\begin{array}{l}\text { RF: deportivas y de } \\
\text { AF }\end{array}$ & $\begin{array}{l}\text { LI: (<3 MET*h-1*día-1) } \\
\text { MO: (3-6 MET*h- } \\
\text { 1*día-1) } \\
\text { VI: (>6 MET*h-1*día-1) } \\
\text { Según cuestionario: } \\
\text { Sedentarios: 1hr por S } \\
\text { Moderadamente acti- } \\
\text { vos: } 1 \text { a 2hrs por S } \\
\text { Activos: 3hrs o más } \\
\text { por S }\end{array}$ & $\begin{array}{l}\text { Los diferentes niveles de } \\
\text { clasificación de AFTL no } \\
\text { varían significativamente } \\
\text { los valores antropométri- } \\
\text { cos o de presión arterial }\end{array}$ \\
\hline
\end{tabular}

H y M: Longitudinal AT: en bicicleta o AFTL: LI: leer, ver tele- MO y VI de AO, RF y RAL

41.053 descriptivo caminando

E: 25-64 (seguimiento RF: montar en bi-

C: sanos

físicamente

(sin historia

e infarto

cerebral

RP: leer, ver la tele-

RA: baile

RF: ciclismo, ca. nasia, correr, jugar útbol, golf, tenis,

RA: bailar
Ausente o LI: inactivo o AFTL de MO o VI se asonos de antropometría parámetros metabólicos minutos de 3 a 5 veces y lipídicos a la $\mathrm{S}$ o ejercicios de-

VI: actividades deportivas al menos 2 veces AT: menos de $15 \mathrm{~min}$ $\min , 30 \min a$ el HDL y negativamente con el LDL, los triglicériRF-RAL: 0 min; aproxi- dos, la CC y el IMC 2-3horas/S; aprox.4-6 MO: (3-6 MET*hSegún cuestionario: Sedentarios: $1 \mathrm{hr}$ por S deradamente actiActivos: 3hrs o más por S 


\begin{tabular}{|c|c|c|c|c|c|}
\hline $\begin{array}{l}\text { Autor/es } \\
\text { (Año) }\end{array}$ & Población & $\begin{array}{c}\text { Diseño del } \\
\text { estudio }\end{array}$ & Tipo de AFTL & Intensidad de AF & Hallazgos \\
\hline 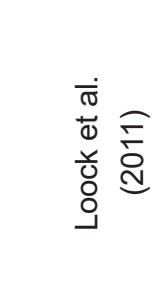 & $\begin{array}{l}\text { M: } 3.542 \\
\text { E: } 30-65 \\
\text { años } \\
\text { C: con } \\
\text { factores de } \\
\text { RCC }\end{array}$ & Transversal & NS & $\begin{array}{l}\text { LI: }\left(<1.000 \mathrm{kcal}^{*} \mathrm{~S}-1\right) \\
\text { MO: }(1.000-1999 \\
\text { kcal*S-1) } \\
\text { VI: }\left(>2.000 \mathrm{kcal}^{*} \mathrm{~S}-1\right)\end{array}$ & $\begin{array}{l}\text { AFTL no alteran signifi- } \\
\text { cativamente los factores } \\
\text { primarios de riesgo para } \\
\text { ECC en M pre- y postme- } \\
\text { nopáusicas }\end{array}$ \\
\hline 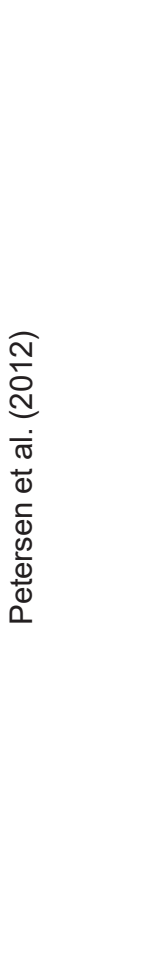 & $\begin{array}{l}\text { H: } 4.487 \text { y } \\
\text { M: } 5.956 \\
\text { C: NS }\end{array}$ & $\begin{array}{l}\text { Longitudinal } \\
\text { (Seguimiento } \\
\text { de } 33 \text { años) }\end{array}$ & $\begin{array}{l}\text { RP: leer, mirar tele- } \\
\text { visión } \\
\text { RF: caminar, montar } \\
\text { en bicicleta, ejercicio } \\
\text { físico, deportes } \\
\text { RAL: jardinería }\end{array}$ & $\begin{array}{l}\text { Sedentario: leer, mirar } \\
\text { televisión, o participar } \\
\text { en AF durante menos } \\
\text { de } 2 \text { horas por S } \\
\text { LI: de } 2 \text { a } 4 \text { h por S } \\
\text { (caminar, montar en } \\
\text { bicicleta, jardinería } \\
\text { ligera, ejercicio físico } \\
\text { ligero) } \\
\text { MO: AF ligera durante } \\
\text { más de } 4 \text { h por S o } \\
\text { vigorosa durante } 2 \text { a } \\
4 \text { h por S (caminar a } \\
\text { paso ligero, ciclismo } \\
\text { rápido, jardinería pe- } \\
\text { sada, deportes que } \\
\text { causan transpiración o } \\
\text { agotamiento) } \\
\text { VI: AF vigorosa durante } \\
\text { más de } 4 \text { horas por S } \\
\text { (ejercicio intenso regu- } \\
\text { lar o deportes varias } \\
\text { veces por S) }\end{array}$ & $\begin{array}{l}\text { H y M que disminuyeron } \\
\text { su participación en AFTL, } \\
\text { aumentaron el riesgo de } \\
\text { IM, en comparación con } \\
\text { las personas que mantu- } \\
\text { vieron la AFTL } \\
\text { Disminuir la participación } \\
\text { en AFTL, se asoció a un } \\
\text { mayor RCC, en compa- } \\
\text { ración con un estilo de } \\
\text { vida físicamente activo } \\
\text { Personas que eran alta- } \\
\text { mente activas físicamen- } \\
\text { te y que permanecieron } \\
\text { así, tuvieron el pronós- } \\
\text { tico más favorable, con } \\
\text { respecto al riesgo de IM }\end{array}$ \\
\hline 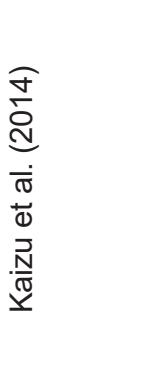 & $\begin{array}{l}\text { H y M: } \\
4.870 \text { E: } \\
\geq 20 \text { años } \\
\text { C: NS }\end{array}$ & Transversal & NS & $\begin{array}{l}\text { Baja intensidad: por } \\
\text { debajo de 3,6 MET } \\
\text { Alta intensidad: por } \\
\text { arriba de 3,6 MET }\end{array}$ & $\begin{array}{l}\text { AFTL se asoció con un } \\
\text { mejor control glucémico y } \\
\text { la mejora de algunos fac- } \\
\text { tores de RCV en pacien- } \\
\text { tes con diabetes tipo } 2 \\
\text { El aumento de AFTL de } \\
\text { mayor intensidad puede } \\
\text { ser apropiado para el } \\
\text { control glucémico }\end{array}$ \\
\hline 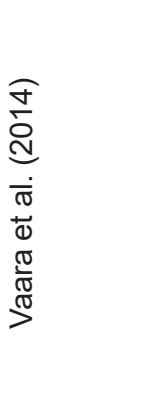 & $\begin{array}{l}\mathrm{H}: 846 \mathrm{E}: \\
25,5 \pm 5,0 \\
\mathrm{C}: \mathrm{NS}\end{array}$ & Transversal & $\begin{array}{l}\text { AFTL: NS } \\
\text { AT } \\
\text { AO }\end{array}$ & $\begin{array}{l}\text { LI: menos de } 1 \text { vez por } \\
\text { S, o no actividades vi- } \\
\text { gorosas, pero AF ligera } \\
\text { o moderada al menos } \\
1 \text { vez a la S } \\
\text { MO: AF vigorosa } 1 \text { o } 2 \\
\text { vez a la S } \\
\text { VI: AF vigorosa } 3 \text { o } 4 \\
\text { veces a la S }\end{array}$ & $\begin{array}{l}\text { La combinación de AFTL } \\
\text { de LI con AFT de LI o AO } \\
\text { de LI se relacionó con } \\
\text { una probabilidad casi } 2 \\
\text { veces mayor de tener } \\
\text { una puntuación alta de } \\
\text { RCV continua, en H adul- } \\
\text { tos jóvenes }\end{array}$ \\
\hline
\end{tabular}

RF: caminar, montar en bicicleta, ejercicio , deportes

LI: de 2 a 4 h por $S$ ligera, ejercicio físico MO: AF ligera durante más de $4 \mathrm{~h}$ por $\mathrm{S}$ vigorosa durante 2 a 4 h por S (caminar a paso ligero, ciclismo causan transpiración o

VI: AF vigorosa durante más de 4 horas por $\mathrm{S}$ veces por S)

Baja intensidad: por

Alta intensidad: por arriba de 3,6 MET
$H$ y $M$ que disminuyeron en AFTL, IM, en comparación con las personas que mantu-

Disminuir la participación AFt, se asoció a un estilo de

Personas que eran altamente activas físicamen- 
NS = no se reporta; ECC = enfermedad cardiaca coronaria; ECV = enfermedad cardiovascular; AFTL = actividad física en el tiempo libre; IMC= índice de masa corporal; $\mathrm{AF}=$ actividad física; $\mathrm{IM}=$ infarto al miocardio; $\mathrm{RCV}=$ riesgo cardiovascular; $\mathrm{RCC}=$ riesgo cardiaco coronario; $\mathrm{H}=$ hombres; $\mathrm{M}=$ mujeres; $\mathrm{C}=$ condición; $\mathrm{E}=$ edad; $\mathrm{HDL}=$ lipoproteínas de alta densidad; $\mathrm{LDL}=$ lipoproteínas de baja densidad; $\mathrm{CC}$ : circunferencia de la cintura; $\mathrm{LI}=$ intensidad ligera; $\mathrm{MO}=$ intensidad moderada; $\mathrm{VI}=$ intensidad vigorosa; $\mathrm{RF}=$ recreación física; RP = recreación de pasatiempos; RAL = recreación al aire libre; $R A$ = recreación artística; $A T$ = actividades físicas de traslado; $\mathrm{AO}=$ actividad física ocupacional; $\mathrm{AH}=$ actividades físicas relacionadas con las labores del hogar; AFT = actividad física total; S = semana.

Fuente: Elaboración propia

Tabla 2. Estudios que examinan la relación entre el tipo y la intensidad de la actividad física realizada en el tiempo libre y la mortalidad por enfermedades cardiovasculares y cardiacas.

\begin{tabular}{|c|c|c|c|c|c|}
\hline $\begin{array}{c}\text { Autor/es } \\
\text { (Año) }\end{array}$ & Población & $\begin{array}{l}\text { Diseño del } \\
\text { estudio }\end{array}$ & Tipo de AFTL & Intensidad de AF & Hallazgos \\
\hline 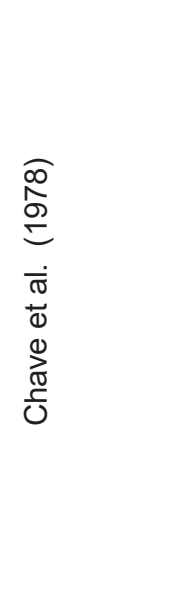 & $\begin{array}{l}\text { H: } 17944 \\
\text { E: } 40-65 \\
\text { años } \\
\text { C: funciona- } \\
\text { rios públicos, } \\
\text { físicamente } \\
\text { sanos }\end{array}$ & $\begin{array}{l}\text { Longitudinal } \\
\text { descriptivo. } \\
\text { (seguimiento } \\
\text { de 7-9 años) }\end{array}$ & $\begin{array}{l}\text { RF: natación, tenis, } \\
\text { caminata, ciclismo, } \\
\text { trote } \\
\text { RAL: escalada, jardi- } \\
\text { nería } \\
\text { Actividades diarias: } \\
\text { caminar, subir esca- } \\
\text { leras, etc. } \\
\text { AH: cavar, cortar ár- } \\
\text { boles, serruchar, mo- } \\
\text { ver muebles pesados, } \\
\text { reemplazar el motor } \\
\text { del carro }\end{array}$ & (7,5 kcal*min o más) & $\begin{array}{l}\mathrm{H} \text { que reportaron ejerci- } \\
\text { cio VI sufrieron menos } \\
\text { muertes por ECC a lo } \\
\text { largo de los años }\end{array}$ \\
\hline 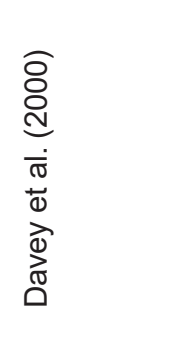 & $\begin{array}{l}\text { H: } 6.702 \\
\text { E: } 40-64 \\
\text { años } \\
\text { C: NS }\end{array}$ & $\begin{array}{l}\text { Longitudinal } \\
\text { (seguimiento } \\
\text { de } 25 \text { años) }\end{array}$ & $\begin{array}{l}\text { RF: natación, ciclis- } \\
\text { mo, atletismo, cami- } \\
\text { nar } \\
\text { RAL: jardinería } \\
\text { AH: mantenimiento } \\
\text { del hogar, trabajo con } \\
\text { madera }\end{array}$ & $\begin{array}{l}\text { Inactivos } \\
\text { Moderadamente ac- } \\
\text { tivos (horticultura, el } \\
\text { mantenimiento de casa } \\
\text { y la carpintería) } \\
\text { Activos (deportes vigo- } \\
\text { rosos durante el tiempo } \\
\text { libre) }\end{array}$ & $\begin{array}{l}\text { La AFTL (RF, RAL), AH } \\
\text { y diarias pueden reducir } \\
\text { el riesgo de mortalidad } \\
\text { por ECC y ECV }\end{array}$ \\
\hline 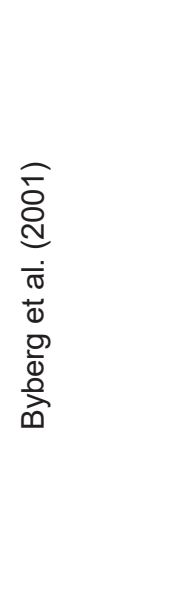 & $\begin{array}{l}\text { H: } 1.860 \\
\text { Edad: } 50 \\
\text { años C: } \sin \\
\text { ECV pre- } \\
\text { existente }\end{array}$ & $\begin{array}{l}\text { Longitudinal } \\
\text { (seguimiento a } \\
\text { los } 20 \text { años) }\end{array}$ & $\begin{array}{l}\text { RP: leer, ver TV, ir al } \\
\text { cine } \\
\text { RF: caminata, ciclis- } \\
\text { mo por placer, de- } \\
\text { portes } \\
\text { RAL: jardinería } \\
\text { Deporte competitivo }\end{array}$ & $\begin{array}{l}\text { Sedentario (Leer, ver } \\
\text { TV, ir al cine) } \\
\text { Moderado (Caminata, } \\
\text { ciclismo por placer) } \\
\text { Regular (Deportes } \\
\text { activos o jardinería } \\
\text { vigorosa por más de } 3 \\
\text { horas a la S) } \\
\text { Atlético (Deporte, } \\
\text { entrenamiento físico } \\
\text { fuerte o deportes com- } \\
\text { petitivos) }\end{array}$ & $\begin{array}{l}\text { La AFTL de alta inten- } \\
\text { sidad y el deporte se } \\
\text { asociaron con un perfil } \\
\text { metabólico más bene- } \\
\text { ficioso y con un menor } \\
\text { riesgo de mortalidad por } \\
\text { ECV, en H }\end{array}$ \\
\hline
\end{tabular}




\begin{tabular}{|c|c|c|c|c|c|}
\hline $\begin{array}{c}\text { Autor/es } \\
\text { (Año) }\end{array}$ & Población & $\begin{array}{c}\text { Diseño del } \\
\text { estudio }\end{array}$ & Tipo de AFTL & Intensidad de AF & Hallazgos \\
\hline 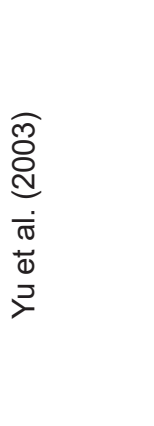 & $\begin{array}{l}\text { H: } 1.975 \\
\text { E: } 49-64 \\
\text { años } \\
\text { C: sin his- } \\
\text { torial clíni- } \\
\text { ca de ECC } \\
\text { al inicio del } \\
\text { estudio }\end{array}$ & $\begin{array}{l}\text { Longitudinal } \\
\text { (seguimiento } \\
\text { de } 11 \text { años) }\end{array}$ & $\begin{array}{l}\text { RF: caminar, jugar bo- } \\
\text { los, golf, nadar, trotar } \\
\text { RAL: andar en velero, } \\
\text { excavar } \\
\text { RA: bailar } \\
\text { AF diarias: subir las } \\
\text { escaleras }\end{array}$ & $\begin{array}{l}\text { LI: caminar, boliche, } \\
\text { andar en velero } \\
\text { MO: golf, excavación, } \\
\text { danza } \\
\text { VI: subir escaleras, } \\
\text { nadar, trotar }\end{array}$ & $\begin{array}{l}\text { La AFTL (RAL, RF, RA) } \\
\text { y AF diarias de VI se } \\
\text { asociaron inversa y sig- } \\
\text { nificativamente con la } \\
\text { mortalidad por ECV y } \\
\text { ECC en H sin historial } \\
\text { de ECC } \\
\text { La AFTL de MO y LI no } \\
\text { se asoció con la mortali- } \\
\text { dad por ECV y ECC }\end{array}$ \\
\hline 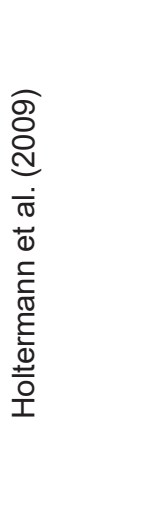 & $\begin{array}{l}\text { H: } 5.249 \\
\text { E: } 40-59 \\
\text { años } \\
\text { C: trabajado- } \\
\text { res caucási- } \\
\text { cos sin pre- } \\
\text { existencia } \\
\text { de ECV }\end{array}$ & $\begin{array}{l}\text { Longitudinal } \\
\text { (seguimiento } \\
\text { de } 30 \text { años) }\end{array}$ & $\begin{array}{l}\text { RP: leer, ir al cine, } \\
\text { ver TV } \\
\text { RF: caminar, bolos, } \\
\text { ciclismo, tenis, tenis } \\
\text { de mesa, bádminton, } \\
\text { natación, fútbol, ba- } \\
\text { lonmano y correr } \\
\text { RAL: jardinería }\end{array}$ & $\begin{array}{l}\text { Sedentarias: LI: ca- } \\
\text { minar, bolos, ciclismo } \\
\text { o AF } \\
\text { durante al menos } 4 \mathrm{hrs} \\
\text { a la S } \\
\text { MO: jardinería, tenis, } \\
\text { tenis de mesa, bádmin- } \\
\text { ton, 3hrs a la S } \\
\text { VI: natación, fútbol, } \\
\text { balonmano y correr, } \\
\text { varias veces por S }\end{array}$ & $\begin{array}{l}\text { AFTL de MO y VI tie- } \\
\text { ne un efecto protector } \\
\text { contra la mortalidad por } \\
\text { enfermedad isquémi- } \\
\text { ca cardíaca en } \mathrm{H} \text { que } \\
\text { tienen niveles bajos o } \\
\text { medios de AF durante } \\
\text { el trabajo }\end{array}$ \\
\hline 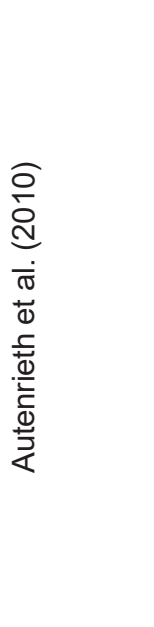 & $\begin{array}{l}\text { H: } 2.373 \text { y } \\
\text { M: } 2.299 \\
\text { E: } 25-74 \\
\text { años } \\
\text { C: NS }\end{array}$ & $\begin{array}{l}\text { Longitudinal } \\
\text { (seguimiento } \\
\text { promedio de } \\
17,8 \text { años) }\end{array}$ & $\begin{array}{l}\text { AT (caminando o bi- } \\
\text { cicleta) } \\
\mathrm{AH}\end{array}$ & $\begin{array}{l}\text { LI: (<3,0 MET) } \\
\text { MO: (3,0-6,0 MET) }\end{array}$ & $\begin{array}{l}\text { AO, AH, AFTL y AFT } \\
\text { disminuyen los riesgos } \\
\text { de mortalidad por ECV } \\
\text { AFTL de } \mathrm{VI} \text {, en compa- } \\
\text { ración con el sedenta- } \\
\text { rismo, reduce significa- } \\
\text { tivamente el riesgo de } \\
\text { mortalidad por ECV } \\
\text { AFTL de LI y MO no se } \\
\text { asociaron con un riesgo } \\
\text { de mortalidad por ECV }\end{array}$ \\
\hline 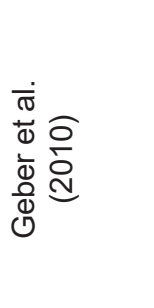 & $\begin{array}{l}\text { Pacientes: } \\
1.521 \\
\mathrm{E}: \leq \text { a } 65 \\
\mathrm{C}: \text { des- } \\
\text { pués de un } \\
\text { primer IM }\end{array}$ & $\begin{array}{l}\text { Longitudinal } \\
\text { (seguimien- } \\
\text { to de 12-13 } \\
\text { años) }\end{array}$ & AFTL: NS & $\begin{array}{l}\text { Categorías: no existen- } \\
\text { te Irregular: menos de } \\
3 \text { sesiones de } 30 \text { minu- } \\
\text { tos por S } \\
\text { Regular: más de } 3 \\
\text { sesiones de } 30 \text { minu- } \\
\text { tos por S }\end{array}$ & $\begin{array}{l}\text { Después de un IM, pa- } \\
\text { cientes regularmente } \\
\text { activos tuvieron cerca } \\
\text { de la mitad del riesgo } \\
\text { de morir, comparados } \\
\text { con pacientes inactivos, } \\
\text { sin importar los hábitos } \\
\text { previos del IM }\end{array}$ \\
\hline
\end{tabular}




\begin{tabular}{|c|c|c|c|c|c|}
\hline $\begin{array}{l}\text { Autor/es } \\
\text { (Año) }\end{array}$ & Población & $\begin{array}{c}\text { Diseño del } \\
\text { estudio }\end{array}$ & Tipo de AFTL & Intensidad de AF & Hallazgos \\
\hline 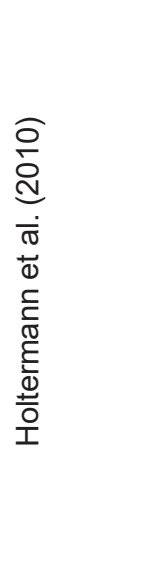 & $\begin{array}{l}\text { H: } 274 \\
\text { E: } 40-59 \\
\text { años C: } \\
\text { empleados } \\
\text { con historia } \\
\text { de ECV }\end{array}$ & $\begin{array}{l}\text { Longitudinal } \\
\text { (seguimiento } \\
\text { de } 30 \text { años) }\end{array}$ & $\begin{array}{l}\text { RP: leer, ver TV, ir al } \\
\text { cine } \\
\text { RF: caminar, ciclismo, } \\
\text { tenis de mesa, bolos, } \\
\text { correr, tenis, fútbol, } \\
\text { bádminton, deportes } \\
\text { competitivos, balon- } \\
\text { mano } \\
\text { RAL: jardinería } \\
\text { AO } \\
\text { AT: caminar }\end{array}$ & $\begin{array}{l}\text { LI: leer, ver TV, ir al } \\
\text { cine } \\
\text { MO: caminar, ciclismo, } \\
\text { jardinería, tenis de } \\
\text { mesa, bolos } \\
\text { VI: correr, tenis, } \\
\text { bádminton deportes } \\
\text { competitivos, fútbol, } \\
\text { balonmano } \\
\text { AO: baja, moderada o } \\
\text { alta según demanda en } \\
\text { el trabajo }\end{array}$ & $\begin{array}{l}\text { No hubo diferencia } \\
\text { significativa entre la in- } \\
\text { tensidad de AFTL (RF- } \\
\text { RP-RAL) y AO, con la } \\
\text { reducción de riesgo por } \\
\text { enfermedad cardíaca } \\
\text { isquémica }\end{array}$ \\
\hline 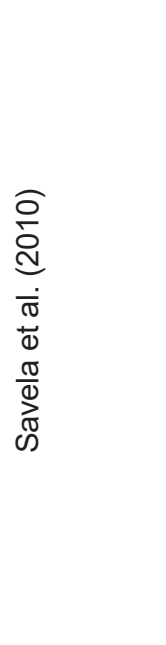 & $\begin{array}{l}\text { H: } 782 \\
\text { E: } 47 \text { años } \\
\text { en prome- } \\
\text { dio } \\
\text { C: inicialmen- } \\
\text { te sanos }\end{array}$ & $\begin{array}{l}\text { Longitudinal } \\
\text { (seguimiento } \\
\text { de } 34 \text { años) }\end{array}$ & $\begin{array}{l}\text { RP: leer, ver TV, ir al } \\
\text { cine } \\
\text { RF: caminar, andar en } \\
\text { bicicleta, bolos, trotar, } \\
\text { correr, nadar, tenis, } \\
\text { bádminton, ejercicio } \\
\text { fuerte, competitivo y } \\
\text { vigoroso } \\
\text { RAL: esquiar, jardine- } \\
\text { ría, ir de pesca, jardi- } \\
\text { nería fuerte }\end{array}$ & $\begin{array}{l}\text { Sedentario: leer, ver } \\
\text { TV, ir al cine } \\
\text { LI: caminar, andar en } \\
\text { bicicleta, esquiar, jardi- } \\
\text { nería, jugar bolos, ir de } \\
\text { pesca } \\
\text { MO: trotar, correr, na- } \\
\text { dar, tenis, bádminton, } \\
\text { jardinería fuerte o ejer- } \\
\text { cicio similar a la S } \\
\text { VI: ejercicio competitivo } \\
\text { y vigoroso de manera } \\
\text { regular o varias veces } \\
\text { a la S }\end{array}$ & $\begin{array}{l}\text { Las muertes por causa } \\
\text { de ECV fueron espe- } \\
\text { cialmente asociadas } \\
\text { con AFTL de menor in- } \\
\text { tensidad: } 45,9 \% \text { ( } n=68 \text { ) } \\
\text { murieron en el grupo de } \\
\text { baja AF, } 37,7 \% \text { ( } n=150 \text { ) } \\
\text { murieron en el grupo de } \\
\text { moderada AF y } 32,6 \% \\
\text { ( } n=77 \text { ) murieron en el } \\
\text { grupo de alta AF }\end{array}$ \\
\hline 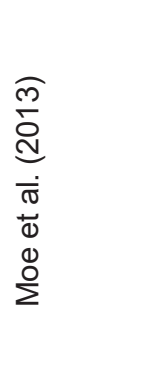 & $\begin{array}{l}\text { H: } 27321 \\
\text { E media: } \\
47,4 \\
\text { M: } 28852 \\
\text { E media } \\
48,5 \\
\text { C: diabéti- } \\
\text { cos }\end{array}$ & $\begin{array}{l}\text { Longitudinal } \\
\text { (seguimiento } \\
\text { de } 24 \text { años) }\end{array}$ & $\begin{array}{l}\text { RAL: pasear, esquiar } \\
\text { RF: nadar, hacer ejer- } \\
\text { cicio, deportes }\end{array}$ & $\begin{array}{l}\text { Físicamente inactivos } \\
0,1-2,9 \text { h de AFTL } \\
\text { por } \mathrm{S} \\
\geq 2 \text { h de AFTL por } \mathrm{S}\end{array}$ & $\begin{array}{l}\text { AFTL se asoció con la } \\
\text { reducción de la morta- } \\
\text { lidad por ECV y cardio- } \\
\text { patía isquémica en per- } \\
\text { sonas con diabetes }\end{array}$ \\
\hline 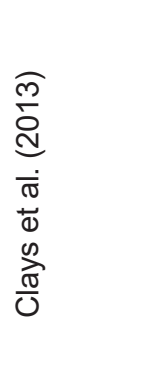 & $\begin{array}{l}\text { H: } 14337 \\
\text { C: } \sin \\
\text { cardiopatía } \\
\text { coronaria al } \\
\text { inicio del } \\
\text { estudio }\end{array}$ & $\begin{array}{l}\text { Estudio de } \\
\text { Cohorte }\end{array}$ & $\begin{array}{l}\text { AFTL } \\
\text { AO }\end{array}$ & $\begin{array}{l}\text { Sin AF semanal } \\
\text { LI: durante la mayoría } \\
\text { de las S } \\
\text { MO o VI: AF intensa } \\
\text { (sudoración y frecuen- } \\
\text { cia del pulso elevada), } \\
\text { durante } 20 \text { min o más, } \\
\text { al menos } 1 \text { vez por S }\end{array}$ & $\begin{array}{l}\text { La AFTL de MO a } \mathrm{VI} \\
\text { se asoció con una tasa } \\
\text { de incidencia coronaria } \\
\text { reducida del } 50 \% \text { en } \mathrm{H} \\
\text { con } \mathrm{AO} \text { de } \mathrm{LI} \text {, pero no } \\
\text { en } \mathrm{H} \text { con } \mathrm{AO} \text { de } \mathrm{VI} \text {, en } \\
\text { donde la tasa de inci- } \\
\text { dencia de eventos coro- } \\
\text { narios fue casi } 4 \text { veces } \\
\text { mayor }\end{array}$ \\
\hline
\end{tabular}




\begin{tabular}{|c|c|c|c|c|c|}
\hline $\begin{array}{c}\text { Autor/es } \\
\text { (Año) }\end{array}$ & Población & $\begin{array}{l}\text { Diseño del } \\
\text { estudio }\end{array}$ & Tipo de AFTL & Intensidad de AF & Hallazgos \\
\hline 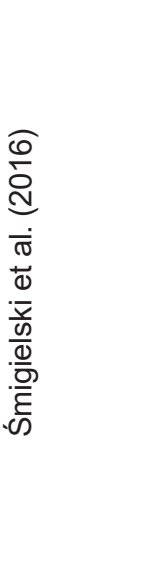 & $\begin{array}{l}\text { H: } 3.577 \\
\text { E: } 50 \text { y } 80 \\
\text { C: NS }\end{array}$ & Transversal & $\begin{array}{l}\text { AT: caminar o ir en } \\
\text { bicicleta hacia / desde } \\
\text { el trabajo } \\
\text { AO } \\
\text { AFTL: NS }\end{array}$ & $\begin{array}{l}\text { Físicamente activos: } \\
\text { caminar o ir en bicicleta } \\
\text { hacia / desde el trabajo } \\
\text { durante al menos } 15 \\
\text { min al día, o realizar } \\
\text { trabajo físico intensivo, } \\
\text { al menos la mitad del } \\
\text { tiempo total de trabajo, } \\
\text { o realizar AF diaria } \\
\text { durante al menos } 30 \\
\text { min, } 4 \text { veces a la S } \\
\text { Sedentarios: falta de } \\
\text { cualquiera de los tipos } \\
\text { de AF descritos antes }\end{array}$ & $\begin{array}{l}\mathrm{H} \text { de } 60-69 \text { años y } 70- \\
80 \text { años de } \mathrm{E} \text { que rea- } \\
\text { lizan AFTL obtienen un } \\
\text { efecto protector contra } \\
\text { ECV y mortalidad de } \\
\text { ECV }\end{array}$ \\
\hline 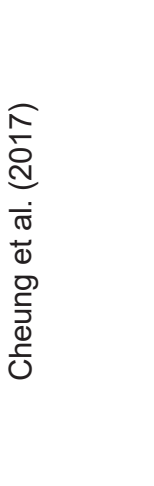 & $\begin{array}{l}\text { H y M: } \\
3.298 \text { de } \\
69 \pm 10 \\
\text { años } \\
\text { C: sin } \\
\text { accidentes } \\
\text { cerebrovas- } \\
\text { culares }\end{array}$ & $\begin{array}{l}\text { Estudio de } \\
\text { Cohorte }\end{array}$ & $\begin{array}{l}\text { RF: Caminar, trotar o } \\
\text { correr, aeróbicos, gim- } \\
\text { nasia, bolos, andar en } \\
\text { bicicleta, nadar o ha- } \\
\text { cer ejercicio acuático, } \\
\text { balonmano, ráquetbol } \\
\text { y squash } \\
\text { RAL: caminatas, } \\
\text { trabajar en el jardín, } \\
\text { equitación } \\
\text { RA: bailar }\end{array}$ & $\begin{array}{l}\text { C de la AFTL: frecuen- } \\
\text { cia de actividad sema- } \\
\text { nal, número de tipos de } \\
\text { actividad (variedad) y } \\
\text { la relación energía-du- } \\
\text { ración (EDR) (energía } \\
\text { promedio por hora) }\end{array}$ & $\begin{array}{l}\text { La frecuencia de AFTL } \\
\text { fue un aspecto protec- } \\
\text { tor contra la mortalidad } \\
\text { ECV en personas adul- } \\
\text { tas mayores }\end{array}$ \\
\hline 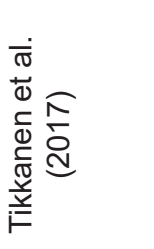 & $\begin{array}{l}\mathrm{H}: 1.136 \text { y } \\
\text { M: } 938 \\
\text { C: diabe- } \\
\text { tes tipo } 1\end{array}$ & $\begin{array}{l}\text { Longitudinal } \\
\text { (seguimiento } \\
\text { de } 10 \text { años) }\end{array}$ & NS & $\begin{array}{l}\text { Sedentario: }<10 \\
\text { MET*h/S } \\
\text { Moderadamente } \\
\text { activo: } 10-40 \mathrm{MET}{ }^{*} \mathrm{~h} / \mathrm{S} \\
\text { Activo: }>40 \mathrm{MET}^{*} \mathrm{~h} / \mathrm{S}\end{array}$ & $\begin{array}{l}\text { AFTL de alta frecuencia } \\
\text { e intensidad, puede re- } \\
\text { ducir el riesgo de even- } \\
\text { tos de ECV en pacien- } \\
\text { tes con diabetes tipo } 1\end{array}$ \\
\hline
\end{tabular}

NS = no se reporta; ECC = enfermedad cardiaca coronaria; ECV = enfermedad cardiovascular; AFTL = actividad física en el tiempo libre; $\mathrm{IMC}=$ índice de masa corporal; $\mathrm{AF}$ = actividad física; $\mathrm{IM}=$ infarto al miocardio; $\mathrm{H}=$ hombres; $\mathrm{M}=$ mujeres; $\mathrm{C}=$ condición; $\mathrm{E}=$ edad; $\mathrm{LI}=$ intensidad ligera; $\mathrm{MO}=$ intensidad moderada; $\mathrm{VI}=$ intensidad vigorosa; $\mathrm{RF}=$ recreación física; $\mathrm{RP}=$ recreación de pasatiempos; $\mathrm{RAL}=$ recreación al aire libre; $\mathrm{RA}=$ recreación artística; $\mathrm{AT}$ = actividades físicas de traslado; $\mathrm{AO}=$ actividad física ocupacional; $\mathrm{AH}=$ actividades físicas relacionadas con las labores del hogar; AFT = actividad física total; $\mathrm{S}$ = semana.

Fuente: Elaboración propia.

Como parte de los resultados se encuentra que no todas las investigaciones analizadas examinaban la intensidad de la actividad física (AF) con los mismos criterios. La mayoría de los estudios señalan la intensidad de la AF por medio de categorías como ligera (LI), moderada (MO) o vigorosa (VI), las cuales fueron establecidas aplicando cuestionarios. En otros, utilizaron mediciones como kcal/ semana o equivalentes metabólicos (MET) y en otras ocasiones fue al evaluar la regularidad o la frecuencia con que realizaban la AF. En consecuencia, al no tener una medida común, se ofrece una breve explicación de los aspectos que 
las personas investigadoras tomaron en cuenta para hacer su clasificación en sus estudios. Por lo tanto, y al igual que en otras investigaciones, las medidas no se estandarizaron, al ser una tarea compleja (10). No obstante, si se desea acceder a un mayor detalle de los criterios que usaron los autores para crear las categorías de la intensidad de cada actividad, se podrá acudir a los estudios en particular.

Por otra parte, en los 29 estudios analizados en esta revisión, se halló que las AFTL no se clasificaban según las categorías existentes en recreación. Por lo tanto, se examinaron las AFTL que se mencionaban en cada estudio y se ordenaron de acuerdo a sus características recreativas. La clasificación se puede apreciar en la columna llamada tipo de AFTL, de las tablas 1 y 2 . Luego del análisis, las categorías de actividades recreativas que surgieron, según la clasificación de la literatura (7), fueron: recreación física, pasatiempos, recreación artística y recreación al aire libre. La mayoría de actividades mencionadas en las investigaciones, se encontraron en la categoría de recreación física. Por otra parte, no todos los estudios explicaban en qué consistían las AFTL. Por ende, no se hicieron las categorías en dichos estudios, pero sí se reportan los resultados como un concepto global de AFTL, el cual podría incluir todos o algunos de los tipos de recreación, establecidos en la literatura.

Finalmente, los resultados mostrados en la Tabla 1 evidencian que, la participación en AFTL se asocia con un menor riesgo ECV y ECC. Solo un estudio indicó que las AFTL no incidían en los factores de riesgo para ECC, pero esto en mujeres pre y postmenopáusicas. Además, varias investigaciones indican que una mayor frecuencia e intensidad en la realización de AFTL es más beneficioso para la prevención de los factores de riesgo de las ECV y ECC. Sin embargo, esto varía según el tipo de población. Con respecto a la mortalidad, en los 14 estudios analizados en la Tabla 2, se encuentra que la participación en las AFTL, y en especial las de alta intensidad, ayudan a disminuir el riego de mortalidad por ECV y ECC.

\section{DISCUSIÓN}

A continuación, se discuten los resultados desde cuatro perspectivas: 1-la clasificación de las AFTL según el tipo de actividad recreativa (AR), 2- la incidencia de las AFTL en los riesgos por ECV y ECC, 3- la incidencia de las AFTL en la mortalidad por ECV y ECC y 4- las recomendaciones para el fomento de las AFTL, que colaboran en la prevención o disminución de los factores de riesgo y mortalidad por ECV y ECC.

\section{AFTL según tipo de AR}

Luego del análisis, las AFTL descritas en cada investigación se clasificaron en recreación física, artística, pasatiempos y al aire libre, identificando así cuatro categorías de las nueve posibles en recreación (7). Las AR, a diferencia de la AF que se hace en el trabajo, en el hogar o para trasladarse hacia el trabajo, se seleccionan voluntariamente y generan gozo y disfrute al participar de ellas. 
Estas características son importantes para incrementar su práctica, porque si las personas sienten deseo de realizarlas pueden entonces obtener gran parte de los beneficios que estas generan en la salud (7).

\section{Incidencia de las AFTL en el riesgo de ECV y ECC, según tipo e intensidad de la AR}

En los diferentes estudios analizados se establece que es importante mantener un estilo de vida físicamente activo durante el tiempo libre, ya que esto permite conservar un índice de masa corporal bajo, poseer parámetros óptimos de lípidos, colesterol, triglicéridos y presión arterial, además de tener relación con un menor índice de fumado, entre otros aspectos que colaboran con un perfil de riesgo cardiovascular (RCV) o riesgo cardiaco coronario ( $R C C$ ) más favorable. Una manera de obtener estos beneficios es por medio de la práctica de AR físicas $(10,11,12,13,14,15,16,17)$. Asimismo, en revisiones previas se ha encontrado que las AFTL se asocian con un menor riesgo de ECC, tanto en $\mathrm{M}$ como en $\mathrm{H}$ de mediana y avanzada edad (10).

Por otra parte, reducir la circunferencia de la cintura (CC) y el índice de masa corporal (IMC) son aspectos que ayudan a prevenir las ECV. Una manera para controlar estos factores, desde la adolescencia hasta la adultez, es por medio de la intensidad y frecuencia con que se realice la AF. La CC llega a ser más pequeña en aquellos adolescentes que participan en AF de intensidad VI, en comparación con los que solo hacen actividades de LI o MO intensidad. Además, los adolescentes que no realizan AFTL aumentan el IMC en mayor medida, en comparación con los que sí participan de manera frecuente en AF. Las mujeres físicamente inactivas, también presentan un aumento en el IMC, con respecto a las que realizan actividades de alta intensidad (18). Sin embargo, la frecuencia o intensidad de la AF no siempre altera los valores antropométricos o la presión arterial en jóvenes (19). No obstante, las asociaciones en ocasiones pueden resultar débiles, debido a la evaluación subjetiva de la AF, por el uso del autoreporte o auto-evaluación, en comparación con medidas objetivas como la aptitud cardiorrespiratoria o aptitud física $(20,21)$.

En pacientes con diabetes tipo 2, también se presenta asociación entre las AFTL con respecto a un mejor control glucémico y con una mejora en algunos factores de RCV (22). Por otra parte, en mujeres pre- y post-menopáusica, la participación en AFTL no altera significativamente los factores de riesgo primarios para la ECC (inactividad física, hipertensión, hipercolesterolemia, obesidad y fumado). Incluso, los factores pueden seguir aumentando con la edad, a pesar de la participación en las AF. Sin embargo, las mujeres físicamente inactivas tienden a presentar mayores riesgos en la salud, que aquellas que participan en los grupos de AF de intensidad MO o VI, lo que implica que las encuestadas pueden minimizar sus riesgos al ser más activas físicamente (23).

Con el paso del tiempo, se ha venido demostrando que tanto las actividades $\mathrm{MO}$ como las $\mathrm{VI}$, incluso las $\mathrm{LI}$ pero realizadas con mayor regularidad, como 
caminar o jardinería, las cuales pueden ser practicadas fácilmente por todas las poblaciones, colaboran en la reducción de RCV y RCC (10). Asimismo, actividades de recreación física como ciclismo, gimnasia, rutinas aeróbicas, patinar, jugar fútbol, baloncesto, balonmano, voleibol, entre otras, o de recreación al aire libre como escalar, o de recreación artística como ballet, bailar, o de pasatiempos como ir de compras, realizadas a una intensidad $\mathrm{MO}, \mathrm{VI}, \mathrm{o} \mathrm{LI}$ pero ejecutadas de manera frecuente, producen beneficios al disminuir los RCV (24). Al respecto, las AR físicas realizadas de forma regular han demostrado mejorar el VO2max y el volumen sistólico, lo que a su vez, provoca efectos positivos en la frecuencia y presión cardiaca, contrarrestando así problemas como los cardiovasculares (25).

En hombres y mujeres con edad promedio de 54 años, se encontró que la AFTL de $\mathrm{MO}$ o $\mathrm{VI}$ intensidad se asoció significativamente con un perfil de RCV más favorable en aspectos de antropometría, parámetros metabólicos y lipídicos. Por ejemplo, se halló que las AFTL de MO intensidad disminuían significativamente los triglicéridos en un $12,3 \%$ y aumentaba el HDL en un $4 \%$. Además, reducían en más del $40 \%$ la posibilidad de tener sobrepeso en comparación con sujetos inactivos. También, protegían ante la posibilidad de tener una mayor CC y triglicéridos elevados y se asociaban inversa y significativamente con la presión arterial diastólica (15). Asimismo, AFTL como ejercicio, caminar, nadar, jardinería, entre otras, llegaron a reducir eventos de ECV en hombres y mujeres, si se realizaban a intensidades VI o $\mathrm{MO}(26,27)$. Con respecto a la intensidad VI, esta se ha asociado con una reducción mayor del $50 \%$ en el riesgo de enfermedad coronaria fatal y no fatal y la intensidad $\mathrm{MO}$ se ha relacionado con un $60 \%$ de disminución en el riesgo de eventos cardiovasculares (27). En otra investigación, se estableció una mayor probabilidad de presentar RCV en hombres jóvenes, cuando ellos combinaban AFTL de intensidad LI con AF de desplazamiento o AF ocupacional de baja intensidad (9).

Por lo tanto, la AFTL realizada en intensidades altas (>6 MET) tiene un efecto sobre el tratamiento de los factores de RCV como el síndrome metabólico, el infarto cerebral, la diabetes, el envejecer, la hipertensión, entre otros. Asimismo, el ejercicio beneficia los niveles de colesterol, sistemas de antioxidantes, presión sanguínea, inflamación y actividad de los canales iónicos (17). No obstante, en el caso de personas obesas primero es necesario fomentar la pérdida de peso, antes de asumir un ejercicio $\mathrm{VI}$ (28). Con respecto a personas adultas mayores, la intensidad LI de la recreación física o de los pasatiempos también resulta beneficiosa, ya que se pueden ejecutar con mayor regularidad y si se realizan casi todos los días, reducen la presión arterial diastólica y la frecuencia cardíaca en reposo en mujeres, así como, el IMC en ambos sexos $(15,24)$.

\section{Incidencia de las AFTL en la mortalidad por ECV y ECC, según tipo e intensidad de la AR}

Los resultados en varios estudios concuerdan con revisiones previas que indican que la participación en las AFTL, incluidas las de recreación física, al aire libre o artísticas, ayudan a disminuir el riesgo de mortalidad por ECV y lo ECC 
$(10,17,29,30)$. Por ejemplo, hombres entre los 60 y 80 años de edad han obtenido un efecto protector contra la mortalidad cardiovascular (MCV), debido a la participación en AFTL en al menos 30 min, 4 veces por semana (31). También, en personas con diabetes se encontró una asociación entre las AFTL con la reducción en la MCV y cardiopatía isquémica. Incluso, el efecto protector del ejercicio físico realizado en el tiempo libre fue mayor, en aquellas personas que informaron estar con tratamiento médico. Entre las razones del por qué esto sucede, está la suma en las mejoras de los factores de RCV, los cuales son más frecuentes en la diabetes que se trata medicamente (32). Las personas que han padecido un infarto al miocardio, también se han beneficiado al mantenerse físicamente activas, por medio de las AFTL, puesto que reducen, en casi la mitad, el riesgo de morir en comparación con aquellas que se mantienen inactivas, llegando a ser esta medida similar a otras de prevención secundaria, como el dejar de fumar o el realizar cambios en la dieta (33).

La participación en AFTL, así como, poseer un nivel alto de aptitud física es importante, porque colabora a reducir el riesgo de mortalidad por enfermedad cardíaca isquémica, al tener un perfil metabólico más beneficioso y al disminuir el riesgo de $\operatorname{MCV}(20,34)$. En cuanto a la intensidad en la que se debe realizar las AFTL, la mayoría de los estudios recomendaron intensidades $\mathrm{MO}$ o VI para su ejecución. Por ejemplo, en una investigación longitudinal realizada con 2373 hombres y 2299 mujeres, con edades entre los 25 y 74 años, se encontró que las AFTL de intensidad VI, en comparación con el sedentarismo, reducían significativamente el riesgo de MCV (35).

En otro caso, hombres de mediana edad que habían reportado realizar ejercicio VI en el tiempo libre, eran los que menos sufrieron muerte por causa de ECC (36). Asimismo, la recreación física, al aire libre o la artística de alta intensidad, se asoció inversa y significativamente con la mortalidad por ECV y ECC, en hombres sin historial por este tipo de enfermedades (30, 34). En trabajadores, las actividades de recreación física o al aire libre, tales como jardinería, deportes como tenis, natación, fútbol, balonmano, entre otras, de intensidad $\mathrm{MO}$ y $\mathrm{VI}$, también generaron efectos protectores contra la mortalidad por enfermedad isquémica cardíaca, en hombres cuyas labores no eran físicamente muy exigentes $(8,37)$. Incluso, en pacientes con diabetes tipo 1, la alta frecuencia e intensidad de las AFTL llegó a reducir el riesgo de eventos de ECV (38).

Las investigaciones demuestran que la AF como la recreación física o al aire libre realizada a altas intensidades, se asocia con una reducción significativa de la MCV, en comparación con la intensidad LI. Además, presentan un efecto significativo en la reducción de la mortalidad por cardiopatía coronaria (10). Al respecto, en un estudio se halló que las muertes causadas por ECV estaban especialmente asociadas con AF de menor intensidad. En ese análisis se encontró que, al realizar AFTL con intensidad VI se daban $32,6 \%(n=77)$ muertes por ECV, en cambio el número de muertes aumentaba a 37,7\% ( $n=150)$ con intensidad $\mathrm{MO}$ y a 45,9\% ( $\mathrm{n=68}$ ) con intensidad LI (39). Los hallazgos de las investigaciones analizadas anteriormente, se asemejan a los encontrados en un 
meta-análisis del 2008, en el cual se determinó que la alta intensidad de AFTL estaba asociada con $27 \%$ menos riesgo de incidencia o mortalidad de ECC, si se le comparaba con los niveles de baja intensidad (40).

\section{Recomendaciones para el fomento de AFTL, que colaboran en prevenir o dis-minuir los factores de riesgo y la mortalidad por ECV y ECC}

Es importante proporcionar desde la infancia una variedad de AFTL, porque según la evidencia, existe una tendencia lineal de disminuir la cantidad de AF a medida que aumenta la edad en ambos sexos (41). Además, la población infantil que es inactiva tiene menos probabilidades de convertirse en adolescentes física-mente activos, si desde su niñez no lo fueron. Por lo que, la realización de AF tem-prana puede predecir el comportamiento en fases posteriores de la vida $(13,42)$.

En personas mayores es fundamental promover su incorporación en programas recreativos y procurar que estos sean estables y duraderos (43). Además, es necesario alentar a la participación constante en AFTL después de la jubilación, debido al aumento del tiempo libre y a las nuevas posibilidades que esto trae consigo (44). También, porque la frecuencia con que se participe en AFTL y la variedad de las mismas, es un aspecto protector contra la mortalidad por ECV en personas adultas mayores (45).

La práctica de AF debe ser regular y sostenida, porque la población que fue físicamente activa pero que se convirtió en sedentaria demuestra un riesgo simi-lar de MCV, que aquella que fue inactiva siempre (10). Por lo tanto, es conveni-ente que profesionales en salud, recreación o educación, o las que están prontas a serlo, alienten activamente a las personas a estar en físicamente en forma, por medio de la realización regular de AF (46). Por otra parte, en pacientes con diabetes, la participación en AFTL de alta intensidad y frecuencia ha resultado beneficiosa, para reducir algunos factores de RCV, disminuir la mortalidad por ECV y cardiopatía isquémica y mejorar el control glucémico $(22,32,38)$.

En las mujeres es preciso hacer conciencia de la importancia de la práctica de AFTL y de la necesidad de aceptar la responsabilidad de realizar AF, como parte importante para su bienestar y salud, porque de esta manera se rompen barreras como la falta de tiempo, de interés, de instalaciones u oportunidades, que le afectan en mayor medida a este sexo (23). Asimismo, el realizar AF al me-nos 150 minutos por semana, se puede ver facilitado si se proveen instalaciones recreativas y acceso a espacios que permitan la práctica de las AFTL, como por ejemplo calles, zonas verdes y aceras en buen estado e iluminadas en la noche, de manera que las personas puedan ejercitarse cuando lo desean y en lugares seguros y habilitados para dicha práctica (47).

En el caso de pacientes que han sufrido un infarto al miocardio y que nunca han sido activos físicamente, es importante que los profesionales en salud enfati-cen en la necesidad de cambiar sus hábitos y realizar AF de manera regular, ya que esta práctica es tan significativa como el dejar de fumar. 
Además, en aquella población que sí acostumbra realizar AFTL, se les debe motivar a mantener ese estilo de vida activo, asegurando que el ejercicio luego de un infarto al miocar-dio es tan seguro como beneficioso (33). Puesto que, convertirse en personas sedentarias, después de haber tenido altos niveles de $A F$, aumenta significati-vamente el riesgo de un infarto al miocardio. Por lo tanto, se debe mantener un estilo de vida físicamente activo durante toda la vida (48).

\section{LIMITACIONES Y CAMINOS FUTUROS}

Una de las limitaciones que presenta esta revisión es que no todos los artículos indicaban cuál era la AF que conformaba el concepto de AFTL. Otra limitante fue que el concepto y medición de la intensidad de las AFTL no era el mismo en todas las investigaciones, por lo que al hablar de intensidad VI, MO y LI se hace de una manera general, tomando en cuenta las distintas especificaciones que señalan los estudios.

Para futuras investigaciones es recomendable profundizar en los resultados que cada tipo de AR puede producir en la prevención de la mortalidad o de los factores de riesgo de ECV y/o ECC. Además, es necesario que un mayor núme-ro de profesionales en recreación se involucren en este tipo de investigación y aporten conocimiento desde su área.

\section{CONCLUSIONES}

De acuerdo con los objetivos planteados al inicio de esta revisión se puede concluir que las AFTL analizadas en las investigaciones se clasificaron en cuatro tipos, recreación física, artística, al aire libre y pasatiempos. De acuerdo con los resultados, la participación en AFTL sí influye positivamente en la prevención de la mortalidad y de los riesgos cardiovasculares y cardíacos, en especial las actividades de recreación física, en algunos casos de recreación al aire libre y en menor medida las de recreación artística. En cuanto a la intensidad, se reco-mienda que sea entre $\mathrm{VI}$ y $\mathrm{MO}$, aunque la LI pero realizada de manera frecuente puede ser beneficiosa para población adulta mayor.

Los resultados pueden variar según las características de cada población, como su edad, condición física, sexo, oficio, patología, entre otros aspectos., sin embargo, las recomendaciones de los estudios aquí analizados ofrecen un punto de referencia acerca de la importancia que tienen las AFTL en la salud y en la prevención de ECV y ECC. Por lo tanto, se enfatiza en la necesidad que las personas valoren y se involucren más en la práctica de las AR. 


\section{REFERENCIAS BIBLIOGRÁFICAS}

1. Fuentes, P. (2010). El problema del sedentarismo en la sociedad actual. Revista Digital, 14(141): 1. Consultado en http://www.efdeportes.com/efd141/el-sedentarismo-en-la-sociedad-actual.htm.

2. Organización Mundial de la Salud (2010). Informe sobre la salud en el mundo. Salud mental: nuevos conocimientos, nuevas esperanzas. Ginebra: OMS.

3. Pacheco, P. (2007). Enfermedades cardiovasculares: Primera causa de muerte en Costa Rica. Medicina vida y salud, 9(6): 4-7.

4. Castillo, L., y Alvarado A. (2006). Enfermedad cardiovascular en Costa Rica. Revista Costarricense de Salud Pública, 15(28): 1409-1429.

5. Garber, C. E., Blissmer, B., Deschenes, M. R., Franklin, B. A., Lamonte, M. J., Lee, I-M., Nieman, D. C., y Swain, D. P. (2011). Quantity and quality of exercise for developing and maintaining cardiorespiratory, musculoskeletal, and neuromotor fitness in apparently healthy adults: Guidance for prescribing exercise. Medicine \& Science in Sports \& Exercise, 43(7): 1334-1359. DOI: 10.1249/MSS.0b013e318213fefb.

6. Vargas, O. (2006). Uso del tiempo libre en el cantón de Turrialba y su influencia en la calidad de vida según edad y género. Revista de Ciencias del Ejercicio y la Salud, 4(1): 51-60.

7. Salazar, C. (2017). Recreación (2da. ed.). San José, Costa Rica: Editorial UCR.

8. Clays, E., De Bacquer, D., Janssens, H., De Clercq, B., Casini, A., Braeckman. L.,... Holtermann, A. (2013). The association between leisure time physical activity and coronary heart disease among men with different physical work demands: A prospective cohort study. European Journal of Epidemiology, N²8: 241-247. DOI: 10.1007/ s10654-013-9764-4.

9. Vaara, J., Kyröläinen, H., Fogelholm, M., Santtila, M., Häkkinen, A., Häkkinen, K.,... Vasankari., T. (2014). Associations of leisure time, commuting, and occupational physical activity with physical fitness and cardiovascular risk factors in young men. Journal of Physical Activity and Health, N¹1: 1482 -1491.

10. Wannamethee, S., y Shaper, A. (2001). Physical activity in the prevention of cardiovascular disease. Sports Med, 31(2): 101-114.

11. Haapanen, N., Miilunpalo, S., Vuori, I., Oja, P., y Pasanen, M. (1997). Association of leisure time physical activity with the risk of coronary heart disease, hypertension and diabetes in middle aged men and women. International Journal of Epidemiology, 26(4): 739-747.

12. Fransson, E., Alfredsson, L., de Faire, U., Knutsson, A., y Westerholm, P. (2003). Leisure time, occupational and household physical activity, adn risk factors for cardiovascular disease in working men and women: the WOLF study. Scand J Public Health, N³1: 324-333.

13. Marti, B. y Vartiainen, E. (1989). Relation between cardiovascular risk factors among 15-year-olds in eastern Finland. Journal of Epidemiology and Community Health, No43: 228-233.

14. SjøI, A., Loft, I., y Jørgensen, T. (2004). Clustering of cardiovascular risk factors. Time trends during 28 years. Scand Cardiovase J, N³8: 222-228. DOI: 10.1080/140174304100163 32. 
15. Sofi, F., Capalbo, A., Marcucci, R., Gori, A., Fedi, S., Macchi, C.,... Gensini, G. (2007). Leisure time but not occupational physical activity significantly affects cardiovascular risk factors in an adult population. European Journal of Clinical Investigation, $N^{\circ} 37$ : 947-953. DOI: 10.1111/j.1365-2362.2007.01884.x.

16. Von Huth, L., Borch, K., y Jørgensen, T. (2007). Commuting physical activity is favourably associated with biological risk factors for cardiovascular disease. Eur J Epidemiol, N²2: 771-779. DOI: 10.1007/s10654-007-9177-3.

17. Yung, L., Laher, I., Yao, X., Yu Chen, Z., Huang, Y., y Leung, F. (2009). Exercise, vascular wall and cardiovascular diseases. Sports Med, 39(1): 45-63.

18. Kujala, M. (2010). Leisure-time physical activity, body mass index, and waist circumference: A longitudinal twin study from adolescence to adulthood. Tesis sin publicar. University of Jyväskylä, Finlandia.

19. Hoyos, I., Irazusta, A., Gravina, L., M. Gil, S., Gil, J., Irazusta, J. (2011). Reduced cardiovascular risk is associated with aerobic fitness in university students. European Journal of Sport Science, 11(2): 87-94.

20. Holtermann, A., Mortensen, O., Burr, H., Søgaard, K., Gyntelberg, F., y Suadicani, P. (2010). Fitness, work, and leisure-time physical activity and ischaemic heart disease and all-cause mortality among men with pre-existing cardiovascular disease. Scand J Work Environ Health, 36(5): 366-372.

21. Lee, D., Sui, X., Ortega, F., Kim, Y., Church, T., Winett, R.,... Blair, S. (2011). Comparisons of leisure-time physical activity and cardiorespiratory fitness as predictors of all-cause mortality in men and women. Sports Med, N45: 504-510.

22. Kaizu, S., Kishimoto, H., Iwase, M., Fujii, H., Ohkuma, T., Ide., H.,... Kitazono, T. (2014). Impact of leisure-time physical activity on glycemic control and cardiovascular risk factors in japanese patients with type 2 diabetes mellitus: The fukuoka diabetes registry. PLOS ONE, 9(6): 1-9. DOI:10.1371/journal.pone.0098768.

23. Loock, H., Wilders, C., Strydom, G., y Ellis, S. (2011). Leisure time physical activity participation in women (30-65 years) with high coronary heart disease risk indicators. African Journal for Physical, Health Education, Recreation and Dance (AJPHERD), 17(4): 624-635.

24. Mensink, G., Ziesea, T., y Kokb, F. (1999). Benefits of leisure-time physical activity on the cardiovascular risk profile at older age. International Journal of Epidemiology, №28: 659-666.

25. Puertas, P., Castro, M., Zurita, F., Chacón R., y Sánchez, M. (2018). La actividad física como medio de prevención de problemas cardiovasculares. Revisión sistemática. Sportis Sci J, 4(3): 443-461. DOI: https://doi.org/10.17979/sportis.2018.4.3.3408.

26. Epstein, L., Miller, G., Stitt, F., y Morris, J. (1976). Vigorous exercise in leisure time, coronary risk-factors, and resting electrocardiogram in middle-aged male civil servants. British Heart Journal, N³8: 403-409.

27. Hu, G., Tuomilehto, J., Borodulin, K., y Jousilahti, P. (2007). The joint associations of occupational, commuting, and leisure-time physical activity, and the Framingham risk score on the 10-year risk of coronary heart disease. European Heart Journal, $\mathrm{N}^{\circ} 28$ : 492-498.

28. Fransson, E., Faire, U., Ahlbom, A., Reuterwall, C., Hallqvist, J., y Alfredsson, L. (2006). The effect of leisure-time physical activity on the risk of acute myocardial in- 
farction depending on body mass index: A population-based case-control study. BMC Public Health, 6(296): 1-9. DOI: 10.1186/1471-2458-6-296.

29. Davey, G., Shipley, M., Batty, G., Morris, J., y Marmot, M. (2000). Physical activity and cause-specific mortality in the Whitehall study. Public Health, N¹14: 308-315.

30. Yu, S., Yarnell, J., Sweetnam, P., y Murray, L. (2003). What level of physical activity protects against premature cardiovascular death? The Caerphilly study. Heart, $\mathrm{N}^{\circ} 89$ : 502-506.

31. Śmigielski, J., Ruszkowska, J., Piotrowski, W., Polakowska, M., Bielecki, W., Hanke, W.,... Drygas, W. (2016). The relationship between physical activity level and selected cardiovascular risk factors and mortality of males $\leq 50$ years in Poland - the results of follow-up of participants of National Multicenter Health Survey WOBASZ. International Journal of Occupational Medicine and Environmental Health, 29(4): 1-16. DOI:10.13075/ijomeh.1896.00660.

32. Moe, B., Augestad, L., y Nilsen, T. (2013). Diabetes severity and the role of leisure time physical exercise on cardiovascular mortality: The nord-trøndelag health study (HUNT), Norway. Cardiovascular Diabetology, 12(83): 1-7.

33. Geber, Y., Myers, V., Goldbourt, U., Benyamini, Y., Scheinowitz, M y Drory, Y. (2010). Long-term trajectory of leisure time physical activity and survival alter first myocardial infarction: population-based cohorte study. European Journal of Epidemiology, $\mathrm{N}^{\circ} 26$ : 109-116. DOI: 10.1007/s10654-010-9523-8.

34. Byberg, L., Zethelius, B., McKeigue, P., y Lithell, H. (2001). Changes in physical activity are associated with changes in metabolic cardiovascular risk factors. Diabetologia. $\mathrm{N}^{\circ} 44:$ 2134-2139.

35. Autenrieth, C., Baumert, J., Baumeister, S., Fischer, B., Peters, A., Do"ring, A.,... Thorand, B. (2010). Association between domains of physical activity anda II cause, cardiovascular and cancer mortality. European Journal of Epidemiology, $\mathrm{N}^{\circ} 26$ : 9199. DOI: 10.1007/s10654-010-9517-6.

36. Chave, W., Morris, N., y Moss, S. (1978). Vigorous exercise in leisure time and the death rate: a study of male civil servants. Journal of Epidemiology and Community Health, N³2: 239-243.

37. Holtermann, A., Mortensen, O., Burr, H., Søgaard, K., Gyntelberg, F., y Suadicani, P. (2009). The interplay between physical activity at work and during leisure time- risk of ischemic heart disease and all cause mortality in middle-aged Caucasian men. Scand J Work Environ Health, 35(6): 466-474.

38. Tikkanen-Dolenc, H., Wadén, J., Forsblom, C., Harjutsalo, V., Thorn, L., Saraheimo, M.,... Groop, P. (2017). Frequent and intensive physical activity reduces risk of cardiovascular events in type 1 diabetes. Diabetologia, 60(3): 574-580. DOI: 10.1007/ s00125-016-4189-8.

39. Savela, S., Koistinen, P., Tilvis, R., Strandberg, A., Pitkälä, K., Salomaa, V.,... Strandberg, T. (2010). Leisure-time physical activity, cardiovascular risk factors and mortality during a 34-year follow-up in men. European Journal of Epidemiology, №25: 619-625. DOI: 10.1007/s10654-010-9483-z.

40. Sofi, S., Capalbo, A., Cesari, F., Abbate, R., \& Gensini, G.F. (2008). Physical activity during leisure time and primary prevention of coronary heart disease: an updated meta-analysis of cohort studies. European Journal of Cardiovascular Prevention and Rehabilitation, 15(3): 247-257. 
41. Martínez, M., Tormo, M., Pérez, D., y Navarro, C. (2003). Actividad física deportiva en una muestra representativa de la población de la Región de Murcia. Gac Sanit, 17(1): 11-9.

42. Dumith, S., Gigante, D., Domingues, M., Hallal, P., Menezes, A., y Kohl, H. (2012). A longitudinal evaluation of physical activity in brazilian adolescents: Tracking, change and predictors. Pediatric Exercise Science, N24: 58-71.

43. Madrigal, J. (2010). Beneficios en la calidad de vida de mujeres entre los 50 y los 81 años de edad al participar en un programa de recreación física grupal. Revista Educación, 34(2): 111-132.

44. Lahti, J., Laaksonen, M., Lahelma, E., y Rahkonen, O. (2011). Changes in leisuretime physical activity after transition to retirement: a follow-up study. International Journal of Behavioral Nutrition and Physical Activity, 8(36): 2-8.

45. Cheung, Y., Moon, Y., Kulick, E., Sacco, R., Elkind, M., y Willey, J. (2017). Leisuretime physical activity and cardiovascular mortality in an elderly population in Northern Manhattan: A prospective cohort study. Journal General Internal Medicine, 32(2): 168-74. DOI: 10.1007/s11606-016-3884-y.

46. Sánchez, G. (2015). Influencia del peso y el nivel de actividad física en la calidad de vida de estudiantes universitarios. Rev. Ib. CC. Act. Fís. Dep, 4(2): 1-8.

47. Hallal, P., Reis, R., Parra, D., Hoehner, C., Brownson, R., y Simoes, E. (2010). Association between perceived environmental attributes and physical activity among adults in Recife, Brazil. Journal of Physical Activity and Health, 7(2): 213-222.

48. Petersen, C., Grønbæk, M., Helge, J., Thygesen, L., Schnohr, P., y Tolstrup, J. (2012). Changes in physical activity in leisure time and the risk of myocardial infarction, ischemic heart disease, and all-cause mortality. European Journal of Epidemiology, 27(2): 91-99. DOI: 10.1007/s10654-012-9656-z. 